\title{
NECESSARY AND SUFFICIENT CONDITIONS FOR A SYSTEM TO BE A QUASIBASIS IN WEIGHTED NORM SPACES
}

\author{
K. S. KAZARIAN \\ Department of Mathematics, C-XV, Universidad Autónoma de Madrid, 28049 Madrid, Spain \\ E-mail: kazaros.kazarian@uam.es \\ and \\ Institute of Mathematics of the National Academy of Sciences of Armenia
}

1. Introduction. Definitions and main results. The following notation and terminology are employed in the sequel. Given a Banach space $B$, we denote by $B^{*}$ its conjugate space. A system $X=\left\{x_{n}: n=1,2, \ldots\right\}$, of elements of $B$, is complete in $B$ if the closure of the set of all finite linear combinations of elements of $X$ coincides with $B$. A system $X=\left\{x_{n}: n=1,2, \ldots\right\}$ is a Schauder basis for $B$ if for every $x$ in $B$, there exists a unique series $\sum_{n=1}^{\infty} a_{n}(x) x_{n}$ that converges to $x$ in the norm of $B$. The existence of a Schauder basis is equivalent to the following [1]: $X$ is complete in $B$ and there exists a conjugate system

$$
X^{*}=\left\{x_{n}^{*}: n=1,2, \ldots\right\} \subset B^{*}
$$

such that

$$
x_{i}^{*}\left(x_{j}\right)=\delta_{i j}, \quad \forall i \in \mathbf{N}, \quad \forall j \in \mathbf{N}
$$

and for all $x$ in $B, \sum_{n=1}^{\infty} y_{n}^{*}(x) x_{n}$ converges to $x$ in the norm of $B$.

Dropping the condition of the unicity of the coefficients, one gets the definition of a quasibasis. More precisely, a system $X=\left\{x_{n}: n=1,2, \ldots\right\}$ is a quasibasis for $B$ if and only if there exists a system $\left\{y_{n}^{*}: n=1,2, \ldots\right\} \subset B^{*}$ such that, for all $x$ in $B$, $\sum_{n=1}^{\infty} y_{n}^{*}(x) x_{n}$ converges to $x$ in the norm of $B$. This generalization of a Schauder basis was introduced by Gelbaum [5] (see also [12]; 278,766).

The system $\left\{y_{n}^{*}: n=1,2, \ldots\right\} \subset B^{*}$ is called an admissible system.

2000 Mathematics Subject Classification: Primary 41A58; Secondary 42C99, 46E30.

Key words and phrases: multiplicative completion, $\Lambda(E)$-spaces, quasibasis.

Research supported by grant BFM2001-0189.

The paper is in final form and no version of it will be published elsewhere. 
Let $\Lambda(E)$ be a Banach space of real valued Lebesgue measurable functions defined on the measurable set $E \subseteq[a, b],-\infty<a<b<+\infty$ with natural linear operations; assume that the measure of $E$ is positive. As usual, we identify functions equal almost everywhere. $\Lambda(E)$ satisfies the following postulates:

(a) if $0 \leq g \leq f$ a.e. on $E$ and $f \in L(E)$, then $g \in L(E)$;

(b) if $f \in \Lambda(E)$ and $\chi_{A}$ is the characteristic function of the set $A$, then $\left\|f \chi_{A}\right\|$ converges to zero as the Lebesgue measure of $A$ (denoted $|A|$ ) converges to zero.

Further in the paper a Lebesgue $\Lambda$-space is a Banach space $\Lambda(E)$ satisfying (a) and (b).

For any measurable subset $F \subset E$, where the measure of $F$ is positive, $\Lambda(F)$ denotes the subspace of $\Lambda(E)$ of functions, identified almost everywhere on $F$, with the norm $\|f\|_{\Lambda(F)}:=\left\|f \chi_{F}\right\|_{\Lambda(E)}$.

The associated space $\Lambda^{\prime}(E)$ of the Lebesgue $\Lambda$-space $\Lambda(E)$ is, by definition, the collection of all Lebesgue measurable functions $\phi$ defined on the set $E$ for which

$$
\|\phi\|_{\Lambda(E)}:=\sup _{\|f\|_{\Lambda(E)}=1} \int_{E} f(t) \phi(t) d t<\infty .
$$

$\Lambda^{\prime}(E)$, just as $\Lambda(E)$, is a Banach lattice and is isometrically isomorphic to $\Lambda^{*}(E)$, the dual of $\Lambda(E)$ (see [10] and also [11]).

MAIN TheOREm. Let $\Lambda(E)$ be a Lebesgue $\Lambda$-space with a quasibasis and let $\left\{f_{n}\right\}_{n=1}^{\infty}$ be a system of measurable a.e. finite functions defined on $E$.

There exists a measurable bounded function $M$ such that $\left\{M f_{n}\right\}_{n=1}^{\infty}$ is a quasibasis in $\Lambda(E)$ if and only if

$\Delta) \quad \forall \epsilon>0 \quad \exists E_{\epsilon}$ such that $\left|E_{\epsilon}\right|>|E|-\epsilon$ and $\left\{f_{n}\right\}_{n=1}^{\infty}$ is a quasibasis in $\Lambda\left(E_{\epsilon}\right)$.

We show that the property $\Delta$ ) is stable with respect to deletion of finitely many elements of the system.

Proposition 1. Let $\left\{f_{n}\right\}_{n=1}^{\infty}$ be a system of measurable a.e. finite functions defined on $E,|E|>0$. If $\left\{f_{n}\right\}_{n=1}^{\infty}$ satisfies the property $\left.\Delta\right)$ then any system obtained from it by deleting finitely many elements also satisfies the same property.

The possibility of obtaining quasibases in weighted $L^{p}, 1 \leq p<\infty$ was observed in [6]. Afterwards in the joint work of the author with R. Zink [7] it was proved that if a system is a quasibasis in an $L^{p_{0}}$ space then the same system is a quasibasis in weighted $L^{p}$ spaces, $1 \leq p \leq p_{0}$, where the weight function that corresponds to the space $L^{p}$ has the form $\omega_{p}=|w|^{p}$. Related questions in the spaces $L(E)$ which satisfy the additional postulates
(c) $L(E) \subset L_{E}^{1}$;
(d) $1 \in L(E)$

have been studied by Ben-Ami Braun [2-4].

2. The Men'shov property. Let $B$ be a Banach space of functions defined on a measurable set $G,|G|>0$. 
Definition 1. We will say that $B$ has the Men'shov property if $\forall \epsilon \exists C(\epsilon)>0$ such that $\forall f \in B$ and for any compact set $\Omega \subset B^{*}$ in the dual space there exists $f^{*} \in B$ that satisfies the following conditions:

$$
\begin{gathered}
\left|\left\{x \in G: f^{*}(x) \neq f(x)\right\}\right| \leq \epsilon ; \quad \operatorname{supp} f:=\{x \in G: f(x) \neq 0\}=\operatorname{supp} f^{*} ; \\
\left\|f^{*}\right\|_{B} \leq C(\epsilon) \cdot\|f\|_{B} ;
\end{gathered}
$$

and

$$
\left|\phi\left(f^{*}\right)\right|<\epsilon \quad \forall \phi \in \Omega .
$$

Theorem 1. Let $\Lambda(E)$ be a Lebesgue $\Lambda$-space. Then $\Lambda(E)$ has the Men'shov property with $C(\epsilon)=\frac{b-a}{\epsilon}$.

Proof. For $\eta \in \mathbf{R}, 0<\eta<|E|$ define a periodic, of period $b-a$, function $h_{\eta}: \mathbf{R} \rightarrow \mathbf{R}$ such that

$$
h_{\eta}(t)= \begin{cases}-\frac{b-a-\eta}{\eta}, & \text { if } t \in[0, \eta] \\ 1, & \text { if } t \in(\eta, b-a) .\end{cases}
$$

It is easy to check that $\int_{0}^{b-a} h_{\eta}(t) d t=0$. One may assume, without loss of generality, that $0<\epsilon<|E|$. Let $f \in \Lambda(E)$ be an arbitrary function and let $\Omega \subset \Lambda^{*}(E)$ be any compact set in the dual space $\Lambda^{*}(E)$. There exists a finite collection of functions $\left\{g_{i}\right\}_{i=1}^{N} \subset \Lambda^{*}(E)$ with the following property: $\forall g \in \Omega \exists j(1 \leq j \leq N)$ such that

$$
\left\|g-g_{j}\right\|_{\Lambda^{*}(E)}<\beta(\epsilon)
$$

where

$$
\beta(\epsilon)=\frac{\epsilon^{2}}{2(b-a)\|f\|_{\Lambda(E)}} .
$$

Employing Fejér's lemma (see [14],49) we get that for any fixed $\eta>0$

$$
\lim _{n \rightarrow \infty} \int_{E} h_{\eta}(n t) f(t) g_{i}(t) d t=0 \quad \text { for all } i(1 \leq i \leq N) .
$$

Let $\eta=\epsilon$. Then we can find $n_{0} \in \mathbf{N}$ such that

$$
\left|\int_{E} h_{\epsilon}\left(n_{0} t\right) f(t) g_{i}(t) d t\right|<\epsilon / 2 \quad \text { for all } i(1 \leq i \leq N) .
$$

Put

$$
f^{*}(t)=h_{\epsilon}\left(n_{0} t\right) f(t) .
$$

By the definition of the function $h_{\eta}$ it follows immediately that $f^{*} \in \Lambda(E)$ and moreover that

We also have that

$$
\left\|f^{*}\right\|_{\Lambda(E)} \leq \frac{b-a}{\epsilon} \cdot\|f\|_{\Lambda(E)} .
$$

$$
\left|\left\{t \in E: f^{*}(t) \neq f(t)\right\}\right| \leq \epsilon .
$$

As for the third condition, one notes that, for every $g \in \Omega$,

$$
\begin{aligned}
\left|\int_{E} f^{*}(t) g(t) d t\right| & \leq\left|\int_{E} f^{*}(t)\left[g(t)-g_{j}(t)\right] d t\right|+\left|\int_{E} f^{*}(t) g_{j}(t) d t\right| \\
& \leq\left\|f^{*}\right\|_{\Lambda(E)} \cdot\left\|g-g_{j}\right\|_{\Lambda^{*}(E)}+\epsilon / 2 \leq \frac{b-a}{\epsilon} \cdot\|f\|_{\Lambda(E)} \cdot \beta(\epsilon)+\epsilon / 2 \leq \epsilon .
\end{aligned}
$$


3. Approximation by truncated polynomials with controlled oscillation. In this section we will show that if a system of functions $\left\{f_{n}\right\}_{n=1}^{\infty}$ satisfies the condition $\Delta)$ then any measurable a.e. finite function defined on the set $E$ can be approximated by truncated polynomials on a subset with measure arbitrarily close to the measure of the set $E$, and the oscillation of the partial sums of the polynomial is controlled by the norm of the function on the subset. In order to approximate a function by a polynomial of the form $\sum_{i=N+1}^{m} a_{i} f_{i}$ one has to modify the function on a set of small measure in such a way that the first $N$ functionals of the admissible system vanish on the modified function. In order to control the oscillation of the partial sums one may divide the given function into pieces with very small norms and then modify each piece. This idea goes back to Talalyan (see [13]). Similar questions in $F$-spaces have been studied in [8].

Theorem 2. Let $\Lambda(E)$ be a Lebesgue $\Lambda$-space, and let $\left\{f_{n}\right\}_{n=1}^{\infty}$ be a system of functions defined on $E$ which satisfies the condition $\Delta)$. If $f \in \Lambda(E)$ then, $\forall \epsilon>0$, and each natural number, $N$, there is a measurable set $G \subset E,|G|>|E|-\epsilon$ and a polynomial, $P=\sum_{k=N}^{M} a_{k} f_{k}$, such that:

$$
\|f-P\|_{L(G)}<\epsilon
$$

and

$$
\sup \left\{\left\|\sum_{k=N}^{s} a_{k} f_{k}\right\|_{L(G)}: N \leq s \leq m\right\}<\epsilon+\|f\|_{L(G)} .
$$

Proof. One may assume, without loss of generality, that $0<\epsilon<|E|$. By virtue of the property $\Delta) \exists E_{1}$ such that $\left|E_{1}\right|>|E|-\epsilon / 2$ and $\left\{f_{n}\right\}_{n=1}^{\infty}$ is a quasibasis in $\Lambda\left(E_{1}\right)$. Let $\left\{g_{n}^{1}\right\}_{n=1}^{\infty} \subset \Lambda^{*}\left(E_{1}\right)$ be an admissible system.

Then, for any $f \in \Lambda\left(E_{1}\right)$ we can define a series such that the $n^{\text {th }}$ partial-sum operator is given by

$$
S_{n}(f)=\sum_{k=1}^{n} g_{k}(\cdot) f_{k}=\sum_{k=1}^{n} a_{k}(f) f_{k}
$$

where

$$
a_{k}(f)=\int_{E_{1}} f(t) g_{k}(t) d t
$$

Since

$$
\lim _{n}\left\|f-S_{n}(f)\right\|_{\Lambda\left(E_{1}\right)}=0, \quad \forall f \in \Lambda\left(E_{1}\right),
$$

an application of the Banach-Steinhaus theorem yields a positive constant, $C$, such that

$$
\left\|S_{n}\right\| \leq C, \quad \forall n \in \mathbf{N} .
$$

There exists a natural number $n$ such that

$$
\left\|f \chi_{\Delta_{n}^{i}}\right\|_{\Lambda\left(E_{1}\right)}<\frac{\epsilon^{2}}{32 \cdot C(b-a)}, \quad i=1, \ldots, n,
$$

where

$$
\Delta_{n}^{i}=E_{1} \cap\left(a+\frac{(i-1)(b-a)}{n}, a+\frac{i(b-a)}{n}\right), \quad i=1, \ldots, n
$$


Among the sets $\left\{\Delta_{n}^{i}\right\}_{i=1}^{n}$ we choose all sets $\left\{E_{1}^{j}\right\}_{j=1}^{n_{0}}$ with positive measure. Evidently

$$
\left|E_{1} \backslash \bigcup_{j=1}^{n_{0}} E_{1}^{j}\right|=0
$$

Thus we have that almost everywhere on $E_{1}$

$$
f=\sum_{j=1}^{n_{0}} f \chi_{E_{1}^{j}}:=\sum_{i=1}^{n_{0}} f^{j} .
$$

Let $n_{1}=N+1$, let

$$
B_{1}=\max \left\{\left\|f_{k}\right\|_{\Lambda\left(E_{1}\right)}: 1 \leq k \leq n_{1}\right\}
$$

and let

$$
\eta_{1}=\frac{\epsilon}{8 n n_{1} B_{1}} .
$$

By virtue of the Men'shov property for the space $\Lambda\left(E_{1}\right)$, there exists a function, $\phi^{1}$, such that

$$
\begin{gathered}
\left|\left\{x \in E_{1}: \phi^{1}(x) \neq f^{1}(x)\right\}\right| \leq \epsilon / 4 n ; \quad \operatorname{supp} f^{1}=\operatorname{supp} \phi^{1} \\
\left\|\phi^{1}\right\|_{\Lambda\left(E_{1}\right)} \leq \frac{4(b-a)}{\epsilon} \cdot\left\|f^{1}\right\|_{\Lambda\left(E_{1}\right)},
\end{gathered}
$$

and

$$
\left.\left|b_{k}^{1}\right|=\mid \int_{E_{1}} \phi^{1}(t) g_{k}(t)\right) d t \mid<\eta_{1}, \quad \forall k \quad\left(1 \leq k \leq n_{1}-1\right) .
$$

The reader should observe that $\operatorname{supp} f^{1} \subset E_{1}^{1}$ and $C(\epsilon / 4 n)=\frac{(b-a)}{n} \cdot \frac{4 n}{\epsilon}=\frac{4(b-a)}{\epsilon}$.

Let $n_{2}$ be a natural number, greater than $n_{1}$, such that

$$
\left\|\phi^{1}-\sum_{k=1}^{n_{2}-1} a_{k}\left(\phi^{1}\right) f_{k}\right\|_{\Lambda\left(E_{1}\right)}<\epsilon \cdot 2^{-n-3},
$$

and let

$$
P_{1}=\sum_{k=n_{1}}^{n_{2}-1} a_{k}\left(\phi^{1}\right) f_{k}
$$

Then,

$$
\begin{aligned}
& \| \phi^{1}- P_{1}\left\|_{\Lambda\left(E_{1}\right)} \leq\right\| \phi^{1}-\sum_{k=1}^{n_{2}-1} a_{k}\left(\phi^{1}\right) f_{k}\left\|_{\Lambda\left(E_{1}\right)}+\right\| \sum_{k=1}^{n_{1}-1} a_{k}\left(\phi^{1}\right) f_{k} \|_{\Lambda\left(E_{1}\right)} \\
&<\epsilon 2^{-n-3}+\left\|\sum_{k=1}^{n_{1}-1} b_{k}^{1} f_{k}\right\|_{\Lambda\left(E_{1}\right)} \leq \epsilon 2^{-n-3}+n_{1} \eta_{1} B_{1}<\epsilon / 4 n .
\end{aligned}
$$

By virtue of (1) we have

$$
\begin{gathered}
\sup \left\{\left\|\sum_{k=n_{1}}^{m} a_{k}\left(\phi^{1}\right) f_{k}\right\|_{\Lambda\left(E_{1}\right)}: n_{1} \leq m<n_{2}\right\} \leq 2 C\left\|\phi^{1}\right\|_{\Lambda\left(E_{1}\right)} \\
\leq 8 C \frac{b-a}{\epsilon} \cdot\left\|f^{1}\right\|_{\Lambda\left(E_{1}\right)} \leq \epsilon / 4
\end{gathered}
$$


Proceeding inductively, suppose that the polynomials associated with the functions $f^{j}, 1 \leq j<i \leq n_{0}<n$, have been determined. Let

$$
B_{i}=\max \left\{\left\|f_{k}\right\|_{\Lambda\left(E_{1}\right)}: 1 \leq k \leq n_{i}\right\},
$$

and let

$$
\eta_{i}=\frac{\epsilon}{8 n n_{i} B_{i}} .
$$

Another application of Men'shov's property yields a function $\phi_{i}$ such that

$$
\begin{gathered}
\left|\left\{x \in E_{i}: \phi^{i}(x) \neq f^{i}(x)\right\}\right| \leq \epsilon / 4 n ; \quad \operatorname{supp} f^{i}=\operatorname{supp} \phi^{i} ; \\
\left\|\phi^{i}\right\|_{\Lambda\left(E_{i}\right)} \leq \frac{4(b-a)}{\epsilon} \cdot\left\|f^{i}\right\|_{\Lambda\left(E_{i}\right)},
\end{gathered}
$$

and

$$
\left.\left|b_{k}^{i}\right|=\mid \int_{E_{1}} \phi^{i}(t) g_{k}(t)\right) d t \mid<\eta_{i}, \quad \forall k \quad\left(1 \leq k \leq n_{i}\right) .
$$

As above, one determines a natural number $n_{i+1}$, greater than $n_{i}$, such that

$$
\left\|\phi^{i}-\sum_{k=1}^{n_{i+1}-1} a_{k}\left(\phi^{i}\right) f_{k}\right\|_{\Lambda\left(E_{1}\right)}<2^{-n-3} \epsilon .
$$

Then, for

$$
P_{i}=\sum_{k=n_{i}}^{n_{i+1}-1} a_{k}\left(\phi^{i}\right) f_{k}
$$

one has

$$
\left\|\phi^{i}-P_{i}\right\|_{\Lambda\left(E_{1}\right)}<2^{-n-3} \epsilon+\eta_{i} n_{i} B_{i}<\epsilon / 4 n,
$$

and

$$
\sup \left\{\left\|\sum_{k=n_{i}}^{m} a_{k}\left(\phi^{i}\right) f_{k}\right\|_{\Lambda\left(E_{1}\right)}: n_{i} \leq k<n_{i+1}\right\} \leq C\left\|\phi^{i}\right\|_{\Lambda\left(E_{1}\right)}<\epsilon / 2 .
$$

Finally, let

$$
P=\sum_{i=1}^{n_{0}} P_{i}, \quad G=\left\{t \in E_{1}: f=\sum_{i=1}^{n_{0}} \phi^{i}\right\}
$$

It is easy to see that

$$
|G|>\left|E_{1}\right|-\sum_{i=1}^{n_{0}} \epsilon / 4 n \geq|E|-\epsilon / 2-\epsilon / 4>|E|-\epsilon .
$$

Since $\sum_{i=1}^{n_{0}} \phi^{i}$ agrees with $f$ on $G$,

$$
\|f-P\|_{L(G)}=\left\|\sum_{i=1}^{n_{0}} \phi^{i}-P\right\|_{L(G)} \leq \sum_{i=1}^{n_{0}}\left\|\phi^{i}-P_{i}\right\|<\epsilon .
$$

As for the third condition, one notes that

$$
\begin{aligned}
\max \left\{\left\|\sum_{i=1}^{j} P_{i}\right\|_{L(G)}: 1 \leq j \leq n_{0}\right\} \leq & \max \left\{\left\|\sum_{i=1}^{j}\left(P_{i}-\phi^{i}\right)\right\|_{L(G)}: 1 \leq j \leq n_{0}\right\} \\
& +\left\|\sum_{i=1}^{j}\left(\phi^{i}-f^{i}\right)\right\|_{L(G)}+\|f\|_{L(G)}
\end{aligned}
$$




$$
<\sum_{i=1}^{j} 2^{-n-2} \epsilon+\|f\|_{L(G)} \leq \epsilon / 2+\|f\|_{L(G)}<\epsilon
$$

thus, for $m \geq N$, say $n_{j} \leq m<n_{j+1}$, one has

$$
\left\|\sum_{k=N}^{m} a_{k} f_{k}\right\|_{L(G)} \leq\left\|\sum_{i=1}^{j} P_{i}\right\|_{L(G)}+\left\|\sum_{k=n_{j}}^{m} a_{k}\left(\phi^{i}\right) f_{k}\right\|_{L(G)}<\epsilon+\|f\|_{L(G)} .
$$

4. Proof of the Main Theorem. The necessity is trivial. Let us prove the sufficiency. Let $\left\{\psi_{i}\right\}_{i=1}^{\infty}$ be a quasibasis in $\Lambda(E)$ with the admissible system $\left\{\psi_{i}^{*}\right\}_{i=1}^{\infty}$. Without loss in generality we can suppose that $\left\|\psi_{i}\right\|_{\Lambda(E)}=1$ for all $i \in \mathbf{N}$. Let $\eta_{i}=2^{-(i+3)}$ for $i \in \mathbf{N}$. The idea of the construction of the weight function $M$ goes back to Price, Zink and Braun [11], [3]. In our case we have to modify the construction because of technical differences.

The weight function $M$ is obtained as an infinite product of functions $M_{k}$ positive almost everywhere and such that each of them is equal to one at a set $D_{k} \subset E$ and is less than one on the complement of $D_{k}$ in $E$. If $\sum_{k=1}^{\infty}\left|E \backslash D_{k}\right|<+\infty$ then, obviously, the function $M=\prod_{k=1}^{\infty} M_{k}$ will be well defined and positive almost everywhere.

Let $\xi \in \Lambda(E),\|\xi\|_{L(E)}=1$, be such that $|\{t \in E: \xi(t) \neq 0\}|=|E|$ and take positive $\delta_{n}<\eta_{n}$ so that

$$
\left\|\psi_{k}\right\|_{L(G)}<\eta_{n} / 4 \quad \forall k(1 \leq k \leq n)
$$

whenever $|G|<\delta_{n}$.

Applying Theorem 1 we find $D_{1} \subset E,\left|D_{1}\right|>|E|-\delta_{1}$ and

$$
P_{11}=\sum_{i=n_{0}(1)+1}^{n_{1}(1)} a_{i} f_{i}, \quad n_{0}(1)=0
$$

such that

$$
\left\|\psi_{1}-P_{11}\right\|_{L\left(D_{1}\right)}<\eta_{1} / 4
$$

and

$$
\sup \left\{\left\|\sum_{k=1}^{s} a_{k} f_{k}\right\|_{L\left(D_{1}\right)}: 1 \leq s \leq n_{1}(1)\right\}<\eta_{1} / 2+\left\|\psi_{1}\right\|_{L\left(D_{1}\right)} .
$$

Let $f_{0}(t)=1$ for $t \in E$ and define

$$
\left[h_{1}(t)\right]^{-1}=\sup _{1 \leq i \leq n_{1}(1)}\left|f_{i}(t)\right| \quad \text { for } \quad t \in E \backslash D_{1} .
$$

Let the function $M_{1}$ be defined, on $E$, by setting

$$
M_{1}(t)=d_{1} \xi(t) \cdot h_{1}(t) \cdot \chi_{E \backslash D_{1}}(t)+\chi_{D_{1}}(t),
$$

where

$$
d_{1}=\eta_{1} / 4 \cdot\left(1+\sup \left\{\left\|\sum_{k=1}^{s} a_{k} f_{k}\right\|_{L\left(D_{1}\right)}: 1 \leq s \leq n_{1}(1)\right\}\right)^{-1} .
$$

We easily check that

$$
\left\|\psi_{1}-M_{1} \cdot P_{11}\right\|_{L\left(D_{1}\right)}<\eta_{1}
$$


and

$$
\sup \left\{\left\|M_{1} \sum_{k=1}^{s} a_{k} f_{k}\right\|_{L(E)}: 1 \leq s \leq n_{1}(1)\right\}<\eta_{1}+\left\|\psi_{1}\right\|_{L(E)} .
$$

Suppose we have constructed polynomials $\left\{P_{j k}\right\}_{j=1, k=j}^{n}$,

$$
P_{j k}=\sum_{i=n_{j-1}(k)+1}^{n_{j}(k)} a_{i} f_{i}
$$

with

$$
\begin{gathered}
1=n_{0}(1)<n_{1}(1)=n_{0}(2)<n_{1}(2)<n_{2}(2)=n_{0}(3)<\ldots \\
<n_{j-1}(j-1)=n_{0}(j)<n_{1}(j)<\ldots<n_{j}(j),
\end{gathered}
$$

and measurable functions $\left\{M_{i}\right\}_{i=1}^{n}, 0<M_{i}(\cdot) \leq 1$, almost everywhere on $E$, such that:

$$
M_{i}(t)=1 \quad \text { if } \quad t \in D_{i} \quad\left|E \backslash D_{i}\right|<\delta_{i} \quad \text { for } \quad 1 \leq i \leq n \text {; }
$$

$$
\left\|\psi_{k}-\prod_{i=1}^{n} M_{i} \sum_{j=k}^{\ell} P_{k j}\right\|_{L(E)} \leq \sum_{j=\ell}^{n} \eta_{j}, \quad \forall k, \quad k \leq \ell \leq n
$$

$$
\sup \left\{\left\|\prod_{i=1}^{n} M_{i} \sum_{i=n_{k-1}(\ell)+1}^{s} a_{i} f_{i}\right\|_{L(E)}: s \leq n_{k}(\ell)\right\} \leq \sum_{j=\ell}^{n} \eta_{j}, \quad \text { if } \quad k<\ell \leq n \text {; }
$$

(4) $\sup \left\{\left\|\prod_{i=1}^{n} M_{i} \sum_{i=n_{k-1}(k)+1}^{s} a_{i} f_{i}\right\|_{L(F)}: n_{k-1}(k)<s \leq n_{k}(k)\right\} \leq \sum_{j=k}^{n} \eta_{j}+\left\|\psi_{k}\right\|_{L(E)}$.

The system $\left\{\prod_{i=1}^{n} M_{i} f_{j}\right\}_{j=1}^{\infty}$ satisfies the condition $\Delta$ ) hence for this system we can successively apply Theorem 2 to the functions

$$
\left\{\psi_{k}-\prod_{i=1}^{n} M_{i} \sum_{j=k}^{n} P_{k j}\right\}_{k=1}^{n} \quad \text { and } \quad \psi_{n+1} .
$$

We can find $D_{n+1} \subset E,\left|D_{n+1}\right|>|E|-\delta_{n+1}$ and

$$
P_{k n+1}=\sum_{i=n_{k-1}(1)+1}^{n_{k}(n+1)} a_{i} f_{i}, \quad n_{0}(n+1)=n_{n}(n)
$$

such that

$$
\begin{gathered}
\left\|\psi_{k}-\prod_{i=1}^{n} M_{i} \sum_{j=k}^{n} P_{k j}-\prod_{i=1}^{n} M_{i} P_{k n+1}\right\|_{L\left(D_{n+1}\right)}<\eta_{n+1} / 4 \\
\left\|\psi_{n+1}-\prod_{i=1}^{n} M_{i} P_{n+1 n+1}\right\|_{L\left(D_{n+1}\right)}<\eta_{n+1} / 4
\end{gathered}
$$

and

$$
\sup \left\{\left\|\prod_{i=1}^{n} M_{i} \sum_{i=n_{k-1}(n+1)+1}^{s} a_{k} f_{k}\right\|_{L\left(D_{n+1}\right)}: 1 \leq s \leq n_{1}(1)\right\}<\eta_{n+1} / 2+\sum_{j=k}^{n} \eta_{j}
$$




$$
\begin{gathered}
\sup \left\{\left\|\sum_{i=n_{n}(n+1)+1}^{s} a_{i} f_{i}\right\|_{L\left(D_{n+1}\right)}: n_{n}(n+1)<s \leq n_{n+1}(n+1)\right\} \\
<\eta_{n+1} / 2+\left\|\psi_{n+1}\right\|_{L\left(D_{n+1}\right)} .
\end{gathered}
$$

Define

$$
\left[h_{n+1}(t)\right]^{-1}=\sup _{0 \leq i \leq n_{n+1}(n+1)}\left|f_{i}(t)\right| \quad \text { for } \quad t \in E \backslash D_{n+1} .
$$

Let the function $M_{n+1}$ be defined, on $E$, by setting

$$
M_{n+1}(t)=d_{n+1} \xi(t) \cdot h_{n+1}(t) \cdot \chi_{E \backslash D_{n+1}}(t)+\chi_{D_{n+1}}(t),
$$

where

$$
d_{n+1}=1 / 4 \eta_{n+1}\left(1+\sum_{\ell}^{n+1} \sum_{k=1}^{\ell} \sup \left\{\left\|\prod_{i=1}^{n} M_{i} \sum_{i=n_{k-1}(\ell)+1}^{s} a_{i} f_{i}\right\|_{L\left(D_{n+1}\right)}: 1 \leq s \leq n_{k}(\ell)\right\}\right)^{-1} \text {. }
$$

Now we easily check that the conditions (2)-(4) hold with $n+1$ instead of $n$.

Let

$$
M(t)=\prod_{i=1}^{\infty} M_{i}(t)
$$

As we have noted before, the function $M$ is well defined and, moreover, $0<M(\cdot) \leq 1$, almost everywhere on $E$. The functions $M_{i}$ have been constructed in such a way that the sequence $\left\{\prod_{i=1}^{n} M_{i}\right\}_{n=1}^{\infty}$ is a fundamental sequence in the space $L_{E}^{\infty}$. Thus in the inequalities $(2)-(4)$ we can pass to the limit and obtain that

$$
\begin{gathered}
\left\|\psi_{k}-M \sum_{j=k}^{\ell} P_{k j}\right\|_{L(E)} \leq 2^{-\ell}, \quad \forall k, \quad k \leq \ell \\
\sup \left\{\left\|M \sum_{i=n_{k-1}(\ell)+1}^{s} a_{i} f_{i}\right\|_{L(E)}: s \leq n_{k}(\ell)\right\} \leq 2^{-\ell}, \quad \text { if } \quad k<\ell \\
\sup \left\{\left\|M \sum_{i=n_{k-1}(k)+1}^{s} a_{i} f_{i}\right\|_{L(F)}: n_{k-1}(k)<s \leq n_{k}(k)\right\} \leq 2^{-k}+\left\|\psi_{k}\right\|_{L(E)} .
\end{gathered}
$$

Let

$$
f_{i}^{*}=a_{i} \psi_{k}^{*}, \quad \text { if } \quad n_{k-1}(\ell)<i \leq n_{k}(\ell), \quad \text { for } \quad \text { some } \quad k \quad \text { and } \quad \ell \geq k .
$$

Then, $\left\{M f_{n}\right\}_{n=1}^{\infty}$ proves to be a quasibasis for $L(E)$ with the admissible system $\left\{f_{n}^{*}\right\}_{n=1}^{\infty}$. The rest of the proof is the same as in [7].

\section{Stability of the $\Delta$ ) property}

Proof of Proposition 1. We may assume, without loss of generality, that the first $N$ elements of $\left\{f_{n}\right\}_{n=1}^{\infty}$ are deleted.

Let $\epsilon>0$ be any positive number. Assume that $0<\epsilon<|E|$. By virtue of the property $\Delta) \exists E_{1}$ such that $\left|E_{1}\right|>|E|-\epsilon / 2$ and $\left\{f_{n}\right\}_{n=1}^{\infty}$ is a quasibasis in $\Lambda\left(E_{1}\right)$. Let $\left\{g_{n}^{1}\right\}_{n=1}^{\infty} \subset \Lambda^{*}\left(E_{1}\right)$ be an admissible system. We may assume, without loss of generality, that $\left\{g_{n}^{1}\right\}_{n=1}^{N}$ are linearly independent in $\Lambda^{*}\left(E_{1}\right)$. Otherwise, we will choose a linearly 
independent collection of elements of the set $\left\{g_{n}^{1}\right\}_{n=1}^{N}$ such that any element from $\left\{g_{n}^{1}\right\}_{n=1}^{N}$ can be represented as a linear combination of the chosen elements.

Let $F_{1}$ be the (measurable) subset of $E_{1}$ which contains those points that are simultaneously points of approximate continuity for each $\left\{g_{n}^{1}\right\}_{n=1}^{N}$. Since each $g_{n}^{1}$ is approximately continuous almost everywhere in $E_{1}$, one has $\left|F_{1}\right|=\left|E_{1}\right|$. One defines, inductively, a set $T=\left\{t_{j}: 1 \leq j \leq N\right\} \subset F_{1}$, on which $\left\{g_{n}^{1}\right\}_{n=1}^{N}$ is linearly independent, in the following manner:

(i) Let $t_{1}$ be any point of $F_{1}$ such that $g_{1}^{1}\left(t_{1}\right) \neq 0$.

(ii) Suppose elements $t_{1}, \ldots, t_{k}$ of $F_{1}$ have been chosen so that $\sum_{j=1}^{k} a_{j} g_{j}^{1}\left(t_{i}\right)=0$, $\forall i=1, \ldots, k$, only if each $a_{j}=0$. Let the numbers $b_{j}$ be chosen (uniquely) so that $f_{k+1}=g_{k+1}^{1}+\sum_{j=1}^{k} b_{j} g_{j}^{1}$ satisfies $f_{k+1}\left(t_{j}\right)=0$, for $1 \leq j \leq k$. Since $\left\{g_{n}^{1}\right\}_{n=1}^{N}$ is linearly independent, $f_{k+1}$ is nonzero on a subset of $F_{1}$ that has positive measure. Let $t_{k+1}$ be any point of the latter set. It follows, at once, that $\left\{\left(g_{j}^{1}\left(t_{1}\right), \ldots, g_{j}^{1}\left(t_{k+1}\right)\right): 1 \leq j \leq k+1\right\}$ is linearly independent. Hence one can find $G_{1} \subset F_{1}, 0<\left|G_{1}\right|<\epsilon / 2$ such that $\left\{g_{n}^{1}\right\}_{n=1}^{N}$ is linearly independent in $\Lambda^{*}\left(G_{1}\right)$. Thus for any collection of real numbers $\left\{c_{n}\right\}_{n=1}^{N}$ one can define a function $\phi \in \Lambda\left(G_{1}\right)$ such that

$$
\int_{G_{1}} \phi(t) g_{n}^{1}(t) d t=c_{n}, \quad 1 \leq n \leq N .
$$

The last condition means that $\forall f \in \Lambda\left(E_{1}\right)$ one can modify the function $f$ on the set $G_{1}$ so that the modified function $f^{*}$ coincides with $f$ on the set $E_{1}^{*}=E_{1} \backslash G_{1},\left|E_{1}^{*}\right|>|E|-\epsilon$ and

$$
\int_{E_{1}} f^{*}(t) g_{n}^{1}(t) d t=0, \quad 1 \leq n \leq N
$$

Hence, $\left\{f_{n}\right\}_{n=N+1}^{\infty}$ is a quasibasis in $\Lambda\left(E_{1}^{*}\right)$.

\section{References}

[1] S. Banach, Theory of Linear Operations, Vol. 2, North-Holland, Amsterdam, 1987.

[2] Ben-Ami Braun, An extension of a result by Talalyan on the representation of measurable functions by Schauder bases, Proc. AMS 34 (1972), 440-446.

[3] Ben-Ami Braun, On the multiplicative completion of certain basic sequences in $L^{p}, 1<$ $p<\infty$, Trans. AMS 176 (1973), 499-508.

[4] Ben-Ami Braun, An extension of a result by Talalyan on the representation of measurable functions by Schauder bases, Proc. AMS 34 (1972), 440-446.

[5] B. R. Gelbaum, Notes on Banach spaces and bases, An. Acad. Brasil 30 (1958), 29-36.

[6] K.S. Kazarian, Closed minimal systems in general separable Banach spaces and in $L^{p}, 1 \leq$ $p<\infty$, Mat. Zametki 53 (1993), 51-61 (in Russian). English transl.: Math Notes 53 (1993), no. 3-4, 279-286.

[7] K. S. Kazarian and R. Zink, Some ramifications of a theorem of Boas and Pollard concerning the completion of a set of functions in $L^{2}$, Trans. AMS 34911 (1997), 4367-4383.

[8] K. S. Kazarian and D. Waterman, Representations of functions by series, Matem. Sbornik 191 (2000), 123-140.

[9] W. A. Luxemburg, Banach function spaces, thesis, Technische Hogeschool te Delft, 1955. 
[10] G. G. Lorentz, Bernstein Polynomials, Mathematical Expositions 8, Univ. of Toronto Press, Toronto, 1953; second edition, Chelsea, New York, 1986.

[11] J. J. Price and R. E. Zink, On sets of functions that can be multiplicatively completed, Ann. Math. 82 (1965), 139-145

[12] I. Singer, Bases in Banach Spaces, II, Springer-Verlag, Berlin, 1981.

[13] A. A. Talalyan, The representation of measurable functions by series, Uspekhi Mat. Nauk 15 (1960), no. 5, 77-142 (Russian); English transl.: Russian Math. Surveys 15 (1960), no. $5,77-136$.

[14] A. Zygmund, Trigonometric Series, 2nd ed., Vol. I, Cambridge Univ. Press, London, 1959. 